\title{
MAXIMUM-ANGLE CONDITION AND TRIANGULAR FINITE ELEMENTS OF HERMITE TYPE
}

\author{
ALEXANDER ŽENÍSEEK
}

\begin{abstract}
Various triangular finite $C^{0}$-elements of Hermite type satisfying the maximum-angle condition are presented and corresponding finite element interpolation theorems are proved. The paper contains also a proof that very general hypotheses due to Jamet are not necessary for such finite elements.
\end{abstract}

\section{INTRODUCTION}

The problem of finite element interpolation theorems under the maximumangle condition was studied in several papers (see [1, 2, 3, 5, 6, 9], [11, pp. 209213]); however, all results concern only triangular finite elements of Lagrange type. (The remark on finite elements of Hermite type in [2, p. 222] is not sufficiently general一see Remark 5.3.)

This note is focused on triangular finite elements of Hermite type which satisfy estimates under the maximum-angle condition and are useful in applications. Our results show that, contrary to finite elements of Lagrange type, sets of parameters uniquely determining such finite elements are different from sets of parameters uniquely determining triangular finite elements which satisfy only the minimum-angle condition.

In $\S \S 2$ and 3 the simplest polynomials of Hermite type, polynomials of third degree, are studied. In both sections nine of ten parameters uniquely determining these polynomials are the same: function values and first derivatives prescribed at the vertices of a triangle. In $\S 2$ the tenth parameter is chosen as the normal derivative at the midpoint of the smallest side of a triangle. This choice enables us to modify and generalize the proof of [14, Theorem 2] in such a way that the estimates for function values do not depend on the geometry of the triangle, and in the estimates for the first derivatives the sine of the minimum angle of the triangle is substituted in the denominator by the sine of the next larger angle of the triangle.

A first version of $\S 2$ was published in [13, Appendix 4]. However, the interpolation result is not introduced there in an optimal form and its proof is

Received by the editor February 26, 1993 and, in revised form, August 19, 1993 and July 5, 1994.

1991 Mathematics Subject Classification. Primary 65N30.

Key words and phrases. Triangular finite elements of Hermite type, maximum- and minimumangle conditions, finite element interpolation theorems.

The work was partly supported by a grant of the European Community under the number ERBCIPA-CT-92-2011/41 OE 1193. 
unnecessarily complicated. Further, the assertion of [13, Remark A.4.13] is not correct; its revision can be found in Propositions 3.4 and 3.5 of this note.

In $\S 3$ we first prove that the finite element from $\S 2$ does not satisfy Jamet's hypotheses [6]. However, by modifying slightly the definition of the tenth parameter, Jamet's theory can be applied.

In the remaining two sections some other possibilities are briefly mentioned.

\section{SOME ELEMENTARY ESTIMATES}

The aim of this section is to prove Theorem 2.1 and its consequences for the finite element interpolation theory (see Theorems 2.7 and 2.8).

2.1. Theorem. Let $\bar{T}$ be a closed triangle with the interior $T$ and vertices $P_{1}, P_{2}, P_{3}$. Let

$$
a=\operatorname{dist}\left(P_{2}, P_{3}\right), \quad b=\operatorname{dist}\left(P_{1}, P_{3}\right), \quad c=\operatorname{dist}\left(P_{1}, P_{2}\right),
$$

and let $\alpha, \beta$ and $\gamma$ be the angles at $P_{1}, P_{2}$ and $P_{3}$, respectively. Let the vertices be denoted in such a way that

$$
a \leq b \leq c, \quad \alpha \leq \beta \leq \gamma .
$$

Let $\varphi \in C^{1}(\bar{T})$, and let

$$
\begin{gathered}
\left|D^{i} \varphi(P)\right| \leq M_{4} \quad \forall|i|=4, \quad \forall P \in T, \\
D^{i} \varphi\left(P_{j}\right)=0 \quad \forall|i| \leq 1(j=1,2,3), \quad \frac{\partial \varphi}{\partial n_{a}}\left(Q_{1}\right)=0,
\end{gathered}
$$

where $Q_{1}$ is the midpoint of the side $P_{2} P_{3}$ and $n_{a}$ the unit normal to $P_{2} P_{3}$. Then

$$
\begin{gathered}
|\varphi(P)| \leq \frac{1}{96}\left(1+4\left(\frac{a}{c}\right)^{3}\right) M_{4} c^{4} \quad \forall P \in \bar{T}, \\
\left|\frac{\partial \varphi}{\partial x_{j}}(P)\right| \leq \frac{4}{15}\left(1+5\left(\frac{a}{c}\right)^{2}\right) \frac{1}{\sin \beta} M_{4} c^{3} \quad \forall P \in \bar{T} \quad(j=1,2) .
\end{gathered}
$$

Theorem 2.1 is a generalization of [14, Theorem 2]. Its proof is based on the following four lemmas.

2.2. Lemma. Let $s_{1}, s_{2}$ be two noncollinear directions making an angle $\omega$. Let $\frac{\partial \varphi}{\partial s_{j}}(P)=k_{j} \quad(j=1,2), P$ being a point of the $\left(x_{1}, x_{2}\right)$-plane. Then

$$
\left|\frac{\partial \varphi}{\partial x_{j}}(P)\right| \leq\left(\left|k_{1}\right|+\left|k_{2}\right|\right) /|\sin \omega| \quad(j=1,2) .
$$

Further, let $s_{1}$ and $s_{2}$ be two directions orthogonal to one another. If $\left|\frac{\partial \psi}{\partial s_{1}}(P)\right| \leq$ $k_{i}(i=1,2)$, then we have for an arbitrary direction $s$

$$
\left|\frac{\partial \psi}{\partial s}(P)\right| \leq\left|k_{1}\right|+\left|k_{2}\right| .
$$

2.3. Lemma. Let $g(0)=\eta_{1}, g(l)=\eta_{2}, g^{\prime}(0)=k_{1}, g^{\prime}(l)=k_{2}$ and $\left|g^{(4)}(s)\right|$ $\leq K_{4}$ in $(0, l)$. Then for $s \in[0, l]$

$$
|g(s)| \leq \max \left|\eta_{j}\right|+\frac{4 l}{27}\left(\left|k_{1}\right|+\left|k_{2}\right|\right)+\frac{K_{4}}{16 \cdot 24} l^{4},
$$




$$
\left|g^{\prime}(s)\right| \leq \frac{3}{2 l}\left(\left|\eta_{1}\right|+\left|\eta_{2}\right|\right)+\max \left|k_{j}\right|+\frac{K_{4}}{24} l^{3} .
$$

Further, if $g(0)=g(l)=g^{\prime}(0)=g^{\prime}(l)=0$, then

$$
\left|g^{\prime \prime}(s)\right| \leq \frac{1}{2} K_{4} l^{2}
$$

2.4. Lemma. Let $g(0)=\eta_{1}, g(l / 2)=\eta_{2}, g(l)=\eta_{3}$ and $\left|g^{(3)}(s)\right| \leq K_{3}$ in $(0, l)$. Then for $s \in[0, l]$

$$
\begin{gathered}
|g(s)| \leq \frac{5}{4} \max \left|\eta_{j}\right|+\frac{\sqrt{3}}{6^{3}} K_{3} l^{3}, \\
\left|g^{\prime}(s)\right| \leq \frac{8}{l} \max \left|\eta_{j}\right|+\frac{1}{4} K_{3} l^{2} .
\end{gathered}
$$

2.5. Lemma. Let $g(0)=\eta_{1}, g(l)=\eta_{2}, g^{\prime}(l)=k_{1}$ and $\left|g^{(3)}(s)\right| \leq K_{3}$ in $(0, l)$. Then for $s \in[0, l]$

$$
|g(s)| \leq \max \left|\eta_{i}\right|+\frac{l}{4}\left|k_{1}\right|+\frac{2}{81} K_{3} l^{3} .
$$

Lemmas 2.2-2.5 are taken from [14] with a modification in (2.6) and improvements in (2.7) and (2.11).

Proof of Theorem 2.1. We restrict our considerations to the case

$$
\left|D^{i} \varphi(P)\right| \leq M_{4} \quad \forall|i|=4, \quad \forall P \in \bar{T} .
$$

In the case (2.2) we can use the trick with an inscribed triangle $\bar{T}^{\prime} \subset T$ in the same way as in [14].

We have by Lemma 2.3 (with $g=\left.\varphi\right|_{P_{2} P_{3}}$ ) and assumptions (2.3) and (2.12)

$$
\begin{gathered}
\left|\left(\left.\varphi\right|_{P_{2} P_{3}}\right)\right| \leq \frac{1}{16 \cdot 24} \cdot 4 M_{4} a^{4}=\frac{1}{96} M_{4} a^{4}, \\
\left|\left(\left.\frac{\partial \varphi}{\partial a}\right|_{P_{2} P_{3}}\right)\right| \leq \frac{1}{24} \cdot 4 M_{4} a^{3}=\frac{1}{6} M_{4} a^{3},
\end{gathered}
$$

where $\partial / \partial a$ denotes the derivative in the direction of $P_{2} P_{3}$. Similarly, Lemma 2.4 with $g=\partial \varphi /\left.\partial n_{a}\right|_{P_{2} P_{3}}$ yields

$$
\left|\left(\left.\frac{\partial \varphi}{\partial n_{a}}\right|_{P_{2} P_{3}}\right)\right| \leq \frac{4 \sqrt{3}}{6^{3}} M_{4} a^{3} .
$$

Using estimates (2.14), (2.15) and Lemma 2.2, we find for an arbitrary direction $s$

$$
\left|\left(\left.\frac{\partial \varphi}{\partial s}\right|_{P_{2} P_{3}}\right)\right| \leq \frac{43}{6^{3}} M_{4} a^{3} \text {. }
$$

Let $P \in \bar{T}, P \neq P_{1}$, and let $B$ be the point of the segment $P_{2} P_{3}$ which lies on the straight line determined by $P_{1}$ and $P$. Setting $l=\operatorname{dist}\left(B, P_{1}\right)$ and considering the function $g=\left.\varphi\right|_{P_{1} B}$, we obtain by means of Lemma 2.3 and (2.3), (2.12), (2.13), (2.16)

$$
|\varphi(P)| \leq \frac{1}{96} M_{4} a^{4}+\frac{4 l}{27} \frac{43}{6^{3}} M_{4} a^{3}+\frac{1}{16 \cdot 24} \cdot 4 M_{4} l^{4},
$$




$$
\left|\frac{\partial \varphi}{\partial s}(P)\right| \leq \frac{3}{2 \cdot 96} M_{4} \frac{a^{4}}{l}+\frac{43}{6^{3}} M_{4} a^{3}+\frac{1}{6} M_{4} l^{3} .
$$

Estimate (2.17) implies (2.4). Estimate (2.18) will be used in deriving (2.5).

Relation (2.8) from Lemma 2.3 with $g=\left.\varphi\right|_{P_{2} P_{3}}$ and relation (2.10) from Lemma 2.4 with $g=\partial \varphi /\left.\partial n_{a}\right|_{P_{2} P_{3}}$ together with assumptions (2.3) yield

$$
\left|\frac{\partial^{2} \varphi}{\partial a^{2}}(B)\right| \leq 2 M_{4} a^{2}, \quad\left|\frac{\partial^{2} \varphi}{\partial a \partial n_{a}}(B)\right| \leq M_{4} a^{2} .
$$

Hence, according to the second part of Lemma 2.2 where we set $\psi=\partial \varphi / \partial a$,

$$
\left|\frac{\partial^{2} \varphi}{\partial a \partial s}(B)\right| \leq 3 M_{4} a^{2} .
$$

Using Lemma 2.5 with $g=\partial \varphi /\left.\partial a\right|_{P_{1} B}$ and taking into account relations (2.3), (2.14), (2.19), we find

$$
\left|\frac{\partial \varphi}{\partial a}(P)\right| \leq \frac{1}{6} M_{4} a^{3}+\frac{3}{4} M_{4} a^{2} l+\frac{8}{81} M_{4} l^{3} .
$$

Inequalities (2.18) and (2.20) together with Lemma 2.2 imply (2.5).

Now we develop some consequences of Theorem 2.1.

2.7. Theorem. A polynomial $p\left(x_{1}, x_{2}\right)$ of degree not greater than three in two variables is uniquely determined by its ten values

$$
D^{i} p\left(P_{j}\right), \quad|i| \leq 1 \quad(j=1,2,3), \quad \frac{\partial p}{\partial n_{a}}\left(Q_{1}\right),
$$

where the meaning of the symbols $P_{i}, Q_{1}$ and $n_{a}$ is the same as in Theorem 2.1 .

Proof. It is sufficient to prove the uniqueness. Let us assume that the values (2.21) are equal to zero. Setting $\varphi\left(x_{1}, x_{2}\right)=p\left(x_{1}, x_{2}\right)$ in Theorem 2.1, we have $M_{4}=0$ and estimate (2.4) implies $p\left(x_{1}, x_{2}\right) \equiv 0$.

2.8. Theorem. Let $u \in C^{1}(\bar{T})$, and let $\left|D^{i} u(P)\right| \leq M_{4}$ for all $|i|=4$ and all $P \in T$. Let $p\left(x_{1}, x_{2}\right)$ be the polynomial of degree not greater than three which satisfies the relations

$$
D^{i} p\left(P_{j}\right)=D^{i} u\left(P_{j}\right), \quad|i| \leq 1 \quad(j=1,2,3), \quad \frac{\partial p}{\partial n_{a}}\left(Q_{1}\right)=\frac{\partial u}{\partial n_{a}}\left(Q_{1}\right)
$$

Then the function

$$
\varphi\left(x_{1}, x_{2}\right) \equiv u\left(x_{1}, x_{2}\right)-p\left(x_{1}, x_{2}\right)
$$

satisfies relations (2.4) and (2.5).

Proof. It follows from the assumptions of Theorem 2.8 that the function (2.23) satisfies all conditions of Theorem 2.1.

It follows from Theorem 2.8 that triangular finite elements with polynomials of third degree uniquely determined by the parameters $(2.21)$ can be used in triangulations satisfying the maximum-angle condition: Estimate (2.5) requires the next-to-smallest angles of all triangles to be bounded away from zero. 
This requirement (we call it the second-angle condition) is equivalent with the maximum-angle condition.

2.9. Remark. The method of the proof of Theorem 2.1 does not work successfully in the case of the classical Hermite triangular finite element of third degree, where the last condition (2.3) is substituted by $\varphi\left(P_{0}\right)=0, P_{0}$ being the center of gravity of $\bar{T}$, because we obtain only $\left|\left(\partial^{2} \varphi /\left.\partial a \partial n_{a}\right|_{P_{2} P_{3}}\right)\right| \leq K M_{4} l^{3} / a \quad(l=$ $\left.\operatorname{dist}\left(P_{1} Q_{1}\right)\right)$ and $l / a \rightarrow \infty$ with $a \rightarrow 0$.

\section{JAMET'S HYPOTHESES. EstimATES IN SOBOLEV NORMS}

First we compare the finite element from $\S 2$ with Jamet's theory [6]. Jamet's hypotheses read as follows:

Let $\mathscr{L}(X, Y)$ denote the set of all linear bounded operators from a normed space $X$ into a normed space $Y$. Let $k, l, m$ be three positive integers, and let $\Pi \in \mathscr{L}\left(W^{l, p}(T), W^{m, p}(T)\right)$, where $p \in[1, \infty]$, be an operator satisfying the following two hypotheses:

(H.1) We have

$$
\Pi u=u \quad \forall u \in P_{k, n},
$$

where $P_{k, n}$ denotes the set of all polynomials in $n$ variables of degree not greater than $k$.

(H.2) There exists a unit vector $\xi$ such that

$$
\frac{\partial u}{\partial \xi}(P)=0 \quad \forall P \in T \quad \Rightarrow \quad \frac{\partial(\Pi u)}{\partial \xi}(P)=0 \quad \forall P \in T .
$$

(We restrict ourselves to this special type of (H.2) because we are interested only in estimates of type (3.3).)

In the case

$$
k=l=3, \quad m=1, \quad n=2, \quad p \in[1, \infty]
$$

it follows from [6, Theorem 2.2]:

3.1. Lemma. Let $\bar{T}$ be the same triangle as in Theorem 2.1 and let us denote $h_{T} \equiv c, \alpha_{T} \equiv \alpha, \beta_{T} \equiv \beta, \gamma_{T} \equiv \gamma$. Let $s_{j k}$ and $s_{j l}$ be the unit vectors parallel to the sides $P_{j} P_{k}$ and $P_{j} P_{l}$, respectively $(j=1,2,3 ; k \neq l)$. Let $\Pi \in \mathscr{L}\left(W^{3, p}(T), W^{1, p}(T)\right)$ be an operator satisfying hypotheses (H.1) and (H.2) for $\xi=s_{j k}$ and $\xi=s_{j l}$. Then we have

$$
|u-\Pi u|_{1, p, T} \leq \frac{C h_{T}^{3}}{\cos \left(\max \left(\vartheta_{j} / 2, \pi / 2-\vartheta_{j} / 2\right)\right)}|u|_{4, p, T} \quad \forall u \in W^{4, p}(T),
$$

where $\vartheta_{1}=\alpha_{T}, \vartheta_{2}=\beta_{T}, \vartheta_{3}=\gamma_{T}$ and $C$ is a constant not depending on $u$ and $T$.

3.2. Remark. In the case $j=3$ the denominator $\cos \left(\max \left(\gamma_{T} / 2, \pi / 2-\gamma_{T} / 2\right)\right)$ can be replaced in (3.3) by $\cos \left(\gamma_{T} / 2\right), \gamma_{T}$ being the maximum angle of $T$ (see Remark in $[6, p .55])$.

In the case $j=2$ the form of the functions $u$ from (3.1) is described in the following lemma. 
3.3. Lemma. Let us use the notation $x \equiv x_{1}, y \equiv x_{2}$. If $\partial u / \partial s_{23} \equiv 0$, then $u(x, y)$ has the form

$$
u(x, y)=F(\eta) \equiv F(\eta(x, y)) \equiv F\left(\left[-\bar{y}_{32}\left(x-x_{2}\right)+\bar{x}_{32}\left(y-y_{2}\right)\right] / J_{2}\right),
$$

where $F(\eta)$ is an arbitrary function differentiable on $[0,1]$ and where

$$
J_{2}=\bar{x}_{32} \bar{y}_{12}-\bar{y}_{32} \bar{x}_{12}
$$

$$
\bar{x}_{j k}=x_{j}-x_{k}, \quad \bar{y}_{j k}=y_{j}-y_{k} \quad(j, k=1,2,3 ; \quad j \neq k) .
$$

If $\partial u / \partial s_{21} \equiv 0$, then

$$
u(x, y)=G(\xi) \equiv G(\xi(x, y)) \equiv G\left(\left[\bar{y}_{12}\left(x-x_{2}\right)-\bar{x}_{12}\left(y-y_{2}\right)\right] / J_{2}\right),
$$

where $G(\xi)$ is an arbitrary function differentiable on $[0,1]$.

Proof. Let $\bar{T}_{0}$ be the triangle lying in the Cartesian coordinate system $(\xi, \eta)$ and having the vertices $R_{1}(0,1), R_{2}(0,0), R_{3}(1,0)$. The transformation

$$
x=x(\xi, \eta) \equiv x_{2}+\bar{x}_{32} \xi+\bar{x}_{12} \eta, \quad y=y(\xi, \eta) \equiv y_{2}+\bar{y}_{32} \xi+\bar{y}_{12} \eta
$$

maps $\bar{T}_{0}$ one-to-one onto $\bar{T}$ in such a way that $R_{j} \leftrightarrow P_{j} \quad(j=1,2,3)$ (for the proof see, for example, [13, Theorem 9.1]). The inverse mapping has the form

$$
\xi=\left[\bar{y}_{12}\left(x-x_{2}\right)-\bar{x}_{12}\left(y-y_{2}\right)\right] / J_{2}, \quad \eta=\left[-\bar{y}_{32}\left(x-x_{2}\right)+\bar{x}_{32}\left(y-y_{2}\right)\right] / J_{2} .
$$

Let us define the function

$$
u^{*}(\xi, \eta)=u(x(\xi, \eta), y(\xi, \eta)) .
$$

Relations (3.8), (3.10) imply

$$
\frac{\partial u^{*}}{\partial \xi}(\xi, \eta)=\frac{\partial u}{\partial x}(x, y) \bar{x}_{32}+\frac{\partial u}{\partial y}(x, y) \bar{y}_{32}=l_{32} \frac{\partial u}{\partial s_{23}}(x, y) \text {. }
$$

Similarly,

$$
\frac{\partial u^{*}}{\partial \eta}(\xi, \eta)=l_{12} \frac{\partial u}{\partial s_{21}}(x, y),
$$

where

$$
l_{j 2}=\sqrt{\bar{x}_{j 2}^{2}+\bar{y}_{j 2}^{2}} \quad(j=1,3) .
$$

If $\partial u / \partial s_{23} \equiv 0$, then, according to (3.11),

$$
\frac{\partial u^{*}}{\partial \xi}(\xi, \eta) \equiv 0
$$

Integrating this relation, we obtain

$$
u^{*}(\xi, \eta)=F(\eta) .
$$

As $u(x, y)=u^{*}(\xi(x, y), \eta(x, y))$, relation (3.14) gives (3.4).

If $\partial u / \partial s_{21} \equiv 0$, then we derive (3.7) by means of (3.12) in a similar way. 
3.4. Proposition. The interpolation operator defined by relations (2.22) satisfies Jamet's hypothesis (H.2) for both $\xi=s_{21}$ and $\xi=s_{23}$ if and only if $\beta=\pi / 2, \beta$ being the angle at $P_{2}$.

Proof. Let the notation be the same as in Lemma 3.3 and its proof, and let us choose the orientation of the unit normal $n_{a}$ to the side $P_{2} P_{3}$ such that $n_{a}=\left(\bar{y}_{32},-\bar{x}_{32}\right) / l_{32}$. Let $\nu_{32}=(\kappa, \lambda)$ be such a vector in the $(\xi, \eta)$-plane that the point $P_{2}+n_{a}$ corresponds one-to-one to the point $R_{2}+\nu_{32}$ in the transformation (3.8). Then we have

$$
\kappa=\left(\bar{x}_{12} \bar{x}_{32}+\bar{y}_{12} \bar{y}_{32}\right) /\left(l_{32} J_{2}\right), \quad \lambda=-l_{32} / J_{2}
$$

and

$$
\kappa=0 \quad \text { if and only if } \beta=\frac{\pi}{2} .
$$

Let $F(\eta)$ be an arbitrary differentiable function on $[0,1]$ and let $q^{*}(\xi, \eta) \in$ $P_{3,2}$ satisfy the conditions

$$
D^{i} q^{*}\left(R_{j}\right)=D^{i} F\left(R_{j}\right),|i| \leq 1(j=1,2,3), \kappa q_{\xi}^{*}\left(S_{1}\right)+\lambda q_{\eta}^{*}\left(S_{1}\right)=\lambda F_{\eta}\left(S_{1}\right),
$$

where the indices $\xi$ and $\eta$ denote the derivatives with respect to $\xi$ and $\eta$, respectively, and where $S_{1}$ is the midpoint of $R_{2} R_{3}$. It is easy to see that conditions (3.17) are satisfied uniquely by the polynomial

$$
\begin{aligned}
q^{*}(\xi, \eta)= & F(0)\left(1-3 \eta^{2}+2 \eta^{3}\right)+F^{\prime}(0)\left(\eta-2 \eta^{2}+\eta^{3}\right) \\
& +F(1)\left(3 \eta^{2}-2 \eta^{3}\right)+F^{\prime}(1)\left(-\eta^{2}+\eta^{3}\right) .
\end{aligned}
$$

Thus, $\partial q^{*} / \partial \xi \equiv 0$ independently of the value of $\kappa$. Hence the polynomial $q(x, y)=q^{*}(\xi(x, y), \eta(x, y))$, where $\xi(x, y), \eta(x, y)$ are the righthand sides of (3.9), satisfies conditions (2.22) with $u(x, y)=F(\eta(x, y))$ and $\partial q / \partial s_{23} \equiv 0$.

Let now $G(\xi)$ be an arbitrary differentiable function on $[0,1]$, and let $p^{*}(\xi, \eta) \in P_{3,2}$ satisfy

$$
D^{i} p^{*}\left(R_{j}\right)=D^{i} G\left(R_{j}\right),|i| \leq 1(j=1,2,3), \kappa p_{\xi}^{*}\left(S_{1}\right)+\lambda p_{\eta}^{*}\left(S_{1}\right)=\kappa G_{\xi}\left(S_{1}\right) .
$$

If $\kappa=0$, then conditions (3.18) are satisfied uniquely by the polynomial

$$
\begin{aligned}
p^{*}(\xi, \eta)= & G(0)\left(1-3 \xi^{2}+2 \xi^{3}\right)+G^{\prime}(0)\left(\xi-2 \xi^{2}+\xi^{3}\right) \\
& +G(1)\left(3 \xi^{2}-2 \xi^{3}\right)+G^{\prime}(1)\left(-\xi^{2}+\xi^{3}\right)
\end{aligned}
$$

and we have $\partial p^{*} / \partial \eta \equiv 0$. Thus, the polynomial $p(x, y)=p^{*}(\xi(x, y), \eta(x, y))$ satisfies conditions $(2.22)$ with $u(x, y)=G(\xi(x, y))$ and $\partial p / \partial s_{21} \equiv 0$.

Let now $\kappa \neq 0$ and let us choose $G(\xi)=\xi^{5}$. Then conditions (3.18) are satisfied uniquely by

$$
p^{*}(\xi, \eta)=-2 \xi^{2}+3 \xi^{3}+\frac{\kappa}{4 \lambda}\left(\xi \eta-\xi^{2} \eta-\xi \eta^{2}\right) .
$$

In this case, $\partial p^{*} / \partial \eta \not \equiv 0$.

The polynomial $p(x, y)=p^{*}(\xi(x, y), \eta(x, y))$, where $p^{*}(\xi, \eta)$ is given by (3.19), satisfies conditions $(2.22)$ with $u(x, y)=\left(\left[\bar{y}_{12}\left(x-x_{2}\right)-\bar{x}_{12}\left(y-y_{2}\right)\right] / J_{2}\right)^{5}$ and we have $\left(\partial p / \partial s_{21}\right)(x, y) \neq 0$ at almost all points $(x, y) \in T$. (It is interesting that in the case $G(\xi)=\xi^{4}$ we have $p^{*}(\xi, \eta)=-\xi^{2}+2 \xi^{3}$.) 
The results obtained in this proof and Lemma 3.3 imply the assertion of Proposition 3.4.

In the case $j=3$ we can prove in a similar way:

3.5. Proposition. The interpolation operator defined by relations (2.22) satisfies Jamet's hypothesis (H.2) for both $\xi=s_{31}$ and $\xi=s_{32}$ if and only if $\gamma=\pi / 2, \gamma$ being the angle at $P_{3}$.

The proof follows similar lines as the proof of Proposition 3.4. Instead of Lemma 3.3 we use the following lemma:

3.6. Lemma. If $\partial u / \partial s_{32} \equiv 0$, then $u(x, y)$ has the form

$$
u(x, y)=F\left(\left[-\bar{y}_{23}\left(x-x_{3}\right)+\bar{x}_{23}\left(y-y_{3}\right)\right] / J_{3}\right),
$$

where $F(\eta)$ is an arbitrary function differentiable on $[0,1]$ and where

$$
J_{3}=\bar{x}_{23} \bar{y}_{13}-\bar{x}_{13} \bar{y}_{23} \text {. }
$$

If $\partial u / \partial s_{31} \equiv 0$, then

$$
u(x, y)=G\left(\left[\bar{y}_{13}\left(x-x_{3}\right)-\bar{x}_{13}\left(y-y_{3}\right)\right] / J_{3}\right),
$$

where $G(\xi)$ is an arbitrary function differentiable on $[0,1]$.

3.7. Remark. a) As $\left|J_{2}\right|=\left|J_{3}\right|$, the set of all functions (3.4) is identical with the set of all functions (3.20).

b) In the proof of Lemma 3.6 the triangle $\bar{T}_{0}$ has the vertices $R_{1}(0,1)$, $R_{2}(1,0), R_{3}(0,0)$ and instead of $(3.8)$ we have

$$
x=x(\xi, \eta) \equiv x_{3}+\bar{x}_{23} \xi+\bar{x}_{13} \eta, \quad y=y(\xi, \eta) \equiv y_{3}+\bar{y}_{23} \xi+\bar{y}_{13} \eta .
$$

Comparing Propositions 3.4 and 3.5 with Theorem 2.8, we see that Jamet's hypothesis (H.2) is not necessary for obtaining pointwise estimates for the gradient under the maximum-angle condition. However, the problem as to how to obtain estimates in the Sobolev norms for $p<\infty$ in the case of the element introduced in $\S 2$, when the sides $P_{1} P_{2}$ and $P_{2} P_{3}$ (or $P_{1} P_{3}$ and $P_{2} P_{3}$ ) are not perpendicular to one another, remains open.

Now we show that modifying slightly the definition of the tenth parameter, we obtain a triangular finite element of Hermite type which satisfies the hypotheses of Jamet's theory.

For every $u \in C^{1}(\bar{T})$ we define $\Pi u \in P_{3,2}$ by the relations (3.23)

$$
D^{i}(\Pi u)\left(P_{j}\right)=D^{i} u\left(P_{j}\right), \quad|i| \leq 1 \quad(j=1,2,3), \quad \frac{\partial(\Pi u)}{\partial s_{21}}\left(Q_{1}\right)=\frac{\partial u}{\partial s_{21}}\left(Q_{1}\right) .
$$

It is easy to see that relations (3.23) define $\Pi u$ uniquely. (If the right-hand sides of $(3.23)$ are equal to zero, then also $\left(\partial(\Pi u) / \partial n_{a}\right)\left(Q_{1}\right)=0$ and $(\Pi u)(x, y) \equiv$ 0 , according to Theorem 2.7.) Thus the operator $\Pi$ satisfies hypothesis (H.1) with $k=3, n=2$. Now we verify that $\Pi$ satisfies also hypothesis (H.2) with $\xi=s_{21}$ and $\xi=s_{23}$.

3.8. Lemma. Let the function $u(x, y)$ be given by (3.4), and let $q \in P_{3,1}$ be the polynomial in one variable $s$ uniquely determined by the conditions

$$
q(0)=F(0), \quad q(1)=F(1), \quad q^{\prime}(0)=F^{\prime}(0), \quad q^{\prime}(1)=F^{\prime}(1) .
$$


Then the polynomial

$$
(\Pi u)(x, y)=q(\eta) \equiv q\left(\left[-\bar{y}_{32}\left(x-x_{2}\right)+\bar{x}_{32}\left(y-y_{2}\right)\right] / J_{2}\right)
$$

satisfies relations (3.23), and we have

$$
\frac{\partial(\Pi u)}{\partial s_{23}}(x, y) \equiv 0 .
$$

3.9. Lemma. Let the function $u$ be given by (3.7), and let $p \in P_{3,1}$ be the polynomial in one variable $s$ uniquely determined by the conditions

$$
p(0)=G(0), \quad p(1)=G(1), \quad p^{\prime}(0)=G^{\prime}(0), \quad p^{\prime}(1)=G^{\prime}(1) .
$$

Then the polynomial

$$
(\Pi u)(x, y)=p(\xi) \equiv p\left(\left[\bar{y}_{12}\left(x-x_{2}\right)-\bar{x}_{12}\left(y-y_{2}\right)\right] / J_{2}\right)
$$

satisfies relations (3.23) and we have

$$
\frac{\partial(\Pi u)}{\partial s_{21}}(x, y) \equiv 0 .
$$

The proof of Lemmas 3.8 and 3.9 follows from the rule of differentiating a composite function.

3.10. Lemma. We have $\Pi \in \mathscr{L}\left(W^{3, p}(T), W^{1, p}(T)\right)$, where the operator $\Pi$ is defined by (3.23) and where $p \in[1, \infty]$.

Proof. The linearity of $\Pi$ is obvious, the boundedness is an easy consequence of the Sobolev imbedding lemma (or, in the case $p=1$, of the fact that $W^{2,1}(T) \subset C(\bar{T})($ see $[10$, p. 300$\left.])\right)$.

Lemmas 3.1, 3.3, 3.8 - 3.10 imply:

3.11. Theorem. Let $u \in W^{4, p}(T)$, where $p \in[1, \infty]$, and let the operator $\Pi$ be defined by (3.23). Then estimate (3.3) holds, i.e.,

$$
|u-\Pi u|_{1, p, T} \leq \frac{C h_{T}^{3}}{\cos \left(\max \left(\beta_{T} / 2, \pi / 2-\beta_{T} / 2\right)\right)}|u|_{4, p, T} .
$$

The following theorem can be obtained similarly:

3.12. Theorem. If we substitute $s_{21}$ in (3.23) by $s_{31}$, then we have for $u \in$ $W^{4, p}(T)$ with $p \in[1, \infty]$

$$
|u-\Pi u|_{1, p, T} \leq \frac{C h_{T}^{3}}{\cos \left(\gamma_{T} / 2\right)}|u|_{4, p, T} .
$$

3.13. Remarks. (a) Besides (3.30) and (3.31) we have, according to [6, Theorem 2.2],

$$
\|u-\Pi u\|_{0, p, T} \leq C h_{T}^{4}|u|_{4, p, T}
$$

In the case $p=2$, estimate (3.32) can be obtained by standard devices (see, e.g., [4]).

(b) The parameters $\left(\partial(\Pi u) / \partial s_{j 1}\right)\left(Q_{1}\right)(i=2,3)$ are parameters of the second kind (for terminology, see [13, p.157]), i.e., they have no influence on the 
global smoothness of a global finite element function defined in a given triangulation. If two such parameters meet at the midpoint of the common side of two adjacent triangles, then they have a common value only by chance.

(c) The parameter $\left(\partial p / \partial n_{a}\right)\left(Q_{1}\right)$ from $\S 2$ is also a parameter of the second kind. Contrary to the parameters $\left(\partial(\Pi u) / \partial s_{j 1}\right)\left(Q_{1}\right)$, it has the advantage that these two parameters have a common value at the midpoint of the common side of two adjacent triangles (when $n_{a}$ is defined for both triangles in the same way); from the point of view of a triangulation they are defined by one parameter. On this common side the global finite element function is not only continuous but it has also continuous first derivatives.

(d) If we substitute the value $\left(\partial \varphi / \partial n_{a}\right)\left(Q_{1}\right)$ by $\left(\partial \varphi / \partial s_{j 1}\right)\left(Q_{1}\right)$ in Theorem 2.1 , then we can derive estimates of the same type (2.4), (2.5). The numerical constants will be only a little different.

3.14. Remark. Let $Q_{2}$ and $Q_{3}$ be the midpoints of the sides $P_{1} P_{3}$ and $P_{1} P_{2}$, respectively. If we substitute $Q_{1}$ and $s_{21}$ in (3.23) by $Q_{3}$ and $s_{23}$, respectively, then estimate (3.30) holds. If we substitute $Q_{1}$ and $s_{21}$ in (3.23) by $Q_{2}$ and $s_{32}$ (or $s_{23}$ ), respectively, then estimate (3.31) holds. In the remaining two cases $Q_{2}, s_{12}$ and $Q_{3}, s_{13}$, we obtain estimates for gradients with $\cos \left(\max \left(\alpha_{T} / 2, \pi / 2-\alpha_{T} / 2\right)\right)$ in the denominator. These two cases are inconvenient in applications when the maximum-angle condition is required for triangulations only.

\section{SOME HIGHER-DEGREE POLYNOMIALS}

In $\S \S 2$ and 3 we modified the classical third-degree Hermite triangular finite element in two different ways and obtained two finite elements permitting estimates under the maximum-angle condition. In this section we generalize these constructions. We shall modify the family introduced by Koukal in [7] and [8].

4.1. Theorem. Let $u \in C^{k}(\bar{T})(k \geq 1)$. A polynomial $p \in P_{2 k+1,2}$ is uniquely determined by the conditions

$$
\begin{gathered}
D^{i} p\left(P_{j}\right)=D^{i} u\left(P_{j}\right), \quad|i| \leq k \quad(j=1,2,3), \\
\frac{\partial^{r} p}{\partial n_{a}^{r}}\left(Q_{j}^{(r)}\right)=\frac{\partial^{r} u}{\partial n_{a}^{r}}\left(Q_{j}^{(r)}\right) \quad(j=1, \ldots, r ; r=1, \ldots, k),
\end{gathered}
$$

where the symbol $\partial / \partial n_{a}$ has the same meaning as in Theorem 2.1 and $Q_{1}^{(r)}, \ldots$, $Q_{r}^{(r)}(1 \leq r \leq k)$ are the points dividing the side $P_{2} P_{3}$ into $r+1$ parts of the same length.

4.2. Theorem. Let $u \in C^{k}(\bar{T})(k \geq 1)$. A polynomial $\Pi u \in P_{2 k+1,2}$ is uniquely determined by the conditions

$$
\begin{gathered}
D^{i}(\Pi u)\left(P_{j}\right)=D^{i} u\left(P_{j}\right), \quad|i| \leq 1 \quad(j=1,2,3), \\
\frac{\partial^{r}(\Pi u)}{\partial s_{31}^{r}}\left(Q_{j}^{(r)}\right)=\frac{\partial^{r} u}{\partial s_{31}^{r}}\left(Q_{j}^{(r)}\right) \quad(j=1, \ldots, r ; r=1, \ldots, k),
\end{gathered}
$$

where $\partial / \partial s_{31}$ denotes the derivative in the direction of the side $P_{3} P_{1}$.

For $k=1$ the assertions of both theorems were proved in $\S \S 2$ and 3 . In the case $k \geq 2$ the proof is a simple modification of the proof of [13, Theorem 17.1], and we omit it.

Generalizing a little the considerations from $\S 3$, we can prove 
4.3. Theorem. Let $u \in W^{2 k+2, p}(T)$, where $k \geq 1$ and $p \in[1, \infty]$, and let the operator $\Pi$ be defined by (4.3), (4.4). Then

$$
|u-\Pi u|_{1, p, T} \leq C \frac{h_{T}^{2 k+1}}{\cos \left(\gamma_{T} / 2\right)}|u|_{2 k+2, p, T} .
$$

4.4. Remarks. (a) In the case $p=2$, standard estimates give

$$
\|u-\Pi u\|_{0, T} \leq C h_{T}^{2 k+2}|u|_{2 k+2, T} .
$$

(b) A generalization of Theorem 2.1 to the case of interpolation polynomials introduced in Theorem 4.1 is possible. Instead of the special Lemmas 2.3-2.5 we can use [12, Theorem 2]. We obtain the estimates

$$
|\varphi(P)| \leq C M_{2 k+2} c^{2 k+2},\left|\frac{\partial \varphi}{\partial x_{j}}(P)\right| \leq \frac{C}{\sin \beta} M_{2 k+2} c^{2 k+1} \quad \forall P \in \bar{T} \quad(j=1,2) .
$$

Details are omitted because they are laborious.

4.5. Remark. The construction of finite elements introduced in Theorem 4.1 implies the following conjecture: It is impossible to construct a triangular finite $C^{1}$-element which satisfies the maximum-angle condition.

\section{SOME REMARKS ON THE CUBIC ELEMENT}

We show by an example that the classical cubic Hermite triangular finite element does not satisfy Jamet's hypothesis (H.2) even in the simplest case.

Let $u(x, y)=y^{4}$, and let the triangle $\bar{T}$ have the vertices $P_{1}(0,0), P_{2}(1,0)$, $P_{3}(0,1)$. Then the polynomial satisfying the first nine conditions $(2.22)$ and condition $p\left(P_{0}\right)=u\left(P_{0}\right)$, where $P_{0}$ is the center of gravity of $\bar{T}$, has the form

$$
p(x, y)=\frac{4}{3}\left(x y-\frac{3}{4} y^{2}-x^{2} y-x y^{2}+\frac{3}{2} y^{3}\right) .
$$

We see that $\frac{\partial u}{\partial x} \equiv 0$ while $\frac{\partial p}{\partial x} \neq 0$ in $T$. Thus, hypothesis (H.2) is not satisfied and we cannot apply Jamet's theory on this finite element. Neither can the estimates of $\S 2$ be modified to this case (see Remark 2.9).

Let us use the same notation as in Theorem 2.1 , and let $P^{*}$ be the point lying on the segment $P_{1} Q_{1}$ and such that $\operatorname{dist}\left(P^{*}, Q_{1}\right)=\varepsilon l$, where $0<\varepsilon \leq \frac{1}{3}$ is fixed and $l=\operatorname{dist}\left(P_{1}, Q_{1}\right)$. (If $P^{*} \equiv P_{0}$, then $\varepsilon=\frac{1}{3}$.)

5.1. Proposition. A polynomial $p \in P_{3,2}$ uniquely determined by the parameters

$$
D^{i} p\left(P_{j}\right),|i| \leq 1(j=1,2,3), \quad p\left(P^{*}\right),
$$

where the point $P^{*}$ is defined above with $\varepsilon=a^{2} /(2 l)^{2}$ permits estimates for the gradient under the maximum-angle condition.

The proof follows similar lines as the proof of [13, Theorem A.4.8] and is omitted.

5.2. Remark. It is clear that a polynomial $p \in P_{3,2}$ from Proposition 5.1 does not satisfy Jamet's hypothesis (H.2).

5.3. Remark. In $[2$, p. 222] the parameters

$$
D^{i} p\left(P_{j}\right), \quad|i| \leq 1 \quad(j=1,2,3), \quad \iint_{T} \frac{\partial^{2} p}{\partial x \partial y} d x d y
$$


were considered in connection with the maximum-angle condition for a cubic triangular finite element on a right triangle with the sides $P_{1} P_{2}$ and $P_{2} P_{3}$ lying on the axes $x$ and $y$, respectively. However, parameters (5.2) do not determine in all cases a polynomial $p \in P_{3,2}$ uniquely. To prove this, let us consider a triangle with vertices $P_{i}\left(x_{i}, y_{i}\right) \quad(i=1,2,3)$, and let $T_{0}$ be the triangle lying in the $(\xi, \eta)$-plane with vertices $P_{1}^{*}(0,0), P_{2}^{*}(1,0), P_{3}^{*}(0,1)$. The transformation

$$
x=x(\xi, \eta) \equiv x_{1}+\bar{x}_{2} \xi+\bar{x}_{3} \eta, \quad y=y(\xi, \eta) \equiv y_{1}+\bar{y}_{2} \xi+\bar{x}_{3} \eta,
$$

where

$$
\bar{x}_{j}=x_{j}-x_{1}, \quad \bar{y}_{j}=y_{j}-y_{1} \quad(j=2,3),
$$

maps the triangle $\bar{T}_{0}$ one-to-one onto $\bar{T}$. Let us set

$$
p^{*}(\xi, \eta)=p(x(\xi, \eta), y(\xi, \eta)) .
$$

If all ten parameters (5.2) are equal to zero, then

$$
\begin{gathered}
D^{i} p^{*}\left(P_{j}^{*}\right)=0 \quad|i| \leq 1 \quad(j=1,2,3) \\
\iint_{T_{0}}\left\{-\bar{x}_{3} \bar{y}_{3} \frac{\partial^{2} p^{*}}{\partial \xi^{2}}+\left(\bar{x}_{2} \bar{y}_{3}+\bar{x}_{3} \bar{y}_{2}\right) \frac{\partial^{2} p^{*}}{\partial \xi \partial \eta}-\bar{x}_{2} \bar{y}_{2} \frac{\partial^{2} p^{*}}{\partial \eta^{2}}\right\} d \xi d \eta=0 .
\end{gathered}
$$

Relations (5.3) imply

$$
p^{*}(\xi, \eta)=K \xi \eta(1-\xi-\eta) .
$$

Inserting (5.5) into (5.4), we obtain

$$
K\left\{2\left(\bar{x}_{2} \bar{y}_{2}+\bar{x}_{3} \bar{y}_{3}\right)-\left(\bar{x}_{2} \bar{y}_{3}+\bar{x}_{3} \bar{y}_{2}\right)\right\}=0 .
$$

If the difference in braces is different from zero, then (5.6) implies $K=0$ and the parameters (5.2) determine uniquely $p \in P_{3,2}$. However, if

$$
2\left(\bar{x}_{2} \bar{y}_{2}+\bar{x}_{3} \bar{y}_{3}\right)=\bar{x}_{2} \bar{y}_{3}+\bar{x}_{3} \bar{y}_{2},
$$

then (5.6) is satisfied with $K \neq 0$, and $p(x, y) \neq 0$ according to (5.5).

Let us describe these situations. We cannot have simultaneously $\bar{x}_{2}=\bar{x}_{3}=0$ (and similarly $\bar{y}_{2}=\bar{y}_{3}=0$ ). Let $\bar{x}_{2} \neq 0$. If $\bar{y}_{2}=0$, then (5.7) gives $\bar{x}_{3}=\bar{x}_{2} / 2$ with arbitrary $\bar{y}_{3} \neq 0$. Conversely, if $\bar{x}_{3}=\bar{x}_{2} / 2$, then (5.7) implies $\bar{y}_{2}=0$. In the other cases,

$$
\bar{y}_{3}=\frac{\left(\bar{x}_{2}-\bar{x}_{3}\right) \bar{y}_{2}}{\bar{x}_{2}-2 \bar{x}_{3}} \quad\left(\bar{y}_{2} \neq 0, \quad \bar{x}_{2} \neq 2 \bar{x}_{3}\right) .
$$

The situation $\bar{x}_{3} \neq 0$ can be treated similarly with the same results.

\section{ACKNOWLEDGMENTS}

The author is very indebted to the referee for his comments, which enabled him to make this paper more concise and the proofs more transparent. 


\section{BIBLIOGRAPHY}

1. T. Apel and M. Dobrowolski, Anisotropic interpolation with applications to the finite element method, Computing 47 (1992), 277-293.

2. I. Babuška and A.K. Aziz, On the angle condition in the finite element method, SIAM J. Numer. Anal. 13 (1976), 214-226.

3. R.E. Barnhill and J.A. Gregory, Sard kernel theorems on triangular domains with applications to finite element error bounds, Numer. Math. 25 (1976), 215-229.

4. J.H. Bramble and M. Zlámal, Triangular elements in the finite element method, Math. Comp. 24 (1970), 809-820.

5. J.A. Gregory, Error bounds for linear interpolation on triangles, Proc. MAFELAP II (J.R. Whiteman, ed.), Academic Press, London, 1976, pp. 163-170.

6. P. Jamet, Estimations d'erreur pour des éléments finis droits presque dégénérés, RAIRO Anal. Numér. 10 (1976), 43-61.

7. S. Koukal, Piecewise polynomial interpolations and their applications in partial differential equations, Sborník VAAZ Brno (1970), 29-30. (Czech)

8. $\ldots$, Piecewise polynomial interpolations in the finite element method, Apl. Mat. 18 (1973), 146-160.

9. M. Křižek, On semiregular families of triangulations and linear interpolation, Appl. Math. $\mathbf{3 6}$ (1991), 223-232.

10. A. Kufner, O. John, and S. Fučí, Function spaces, Academia, Prague, 1977.

11. J.L. Synge, The hypercircle in mathematical physics, Cambridge Univ. Press, London, 1957.

12. A. Ženíšek, Interpolation polynomials on the triangle, Numer. Math. 15 (1970), 283-296.

13. _ Nonlinear elliptic and evolution problems and their finite element approximation, Academic Press, London, 1990.

14. M. Zlánal, On the finite element method, Numer. Math. 12 (1968), 394-409.

Department of Mathematics, Technical University, Technická 2, 61669 Brno, Czech REPUBLIK

E-mail address: zenisek@kinf.fme.vutbr.cz 